Biologie Aujourd'hui, 207 (3), 151 (2013)

(C) Société de Biologie, 2013

DOI: $10.1051 /$ jbio/2013020

\title{
Hommage à Jacques Polonovski
}

Jacques Polonovski, Professeur de Biochimie et de Biologie Moléculaire à l'Université Pierre et Marie Curie, s'est éteint le 7 octobre 2013 à 94 ans.

Après des études à l'École Normale Supérieure puis à la Faculté de Médecine de Paris, il commença une carrière de recherche scientifique dans le laboratoire du Professeur Machebeuf où il fut l'un des acteurs de la découverte des cénapses lipidoprotéidiques, ancêtres des lipoprotéines. Nommé Maître de Conférences à la Faculté de Médecine puis Professeur titulaire de chaire, il participa en 1963 à la création du premier CHU parisien, le CHU Saint-Antoine, qui regroupait autour de la Faculté de Médecine de la rue de Chaligny les Hôpitaux Saint-Antoine, Tenon, Rothschild et Trousseau.

Il y créa une unité de recherche associée au CNRS dédiée au métabolisme des lipides et des phospholipases qu'il dirigea jusqu'en 198\%. De son laboratoire sont issus une bonne douzaine de professeurs en biochimie et biologie moléculaire ainsi qu'en biologie cellulaire tant en Ile-de-France qu'en Région. Il créa également le laboratoire de biochimie spécialisé à l'Hôpital Saint-Antoine qu'il dirigea jusqu'à sa retraite.

Qu'il nous soit permis d'ajouter à ces lignes de Gilbert Béréziat, qui fut son collaborateur puis son successeur, l'hommage d'un des anciens étudiants de Jacques Polononovski. En effet, dans les années 60, celui-ci intervenait dans la préparation à l'agrégation de Physiologie-Biochimie de l'ENSET et, le samedi matin, personne n'aurait manqué ses leçons de biochimie métabolique à Saint-Antoine, qui associaient un savoir apparemment inépuisable à une extraordinaire clarté d'expression, rendant assimilable et presque attrayante cette matière aride. Ces remarquables qualités didactiques se retrouvaient dans le manuel qu'il avait repris, avec plusieurs co-auteurs, au décès de son père, lui-même biochimiste et victime, avec sa mère, d'un accident de voiture. Régulièrement mis à jour, le « Polo» a été la bible des étudiants biologistes et même des chercheurs jusqu'à l'avènement de la biologie moléculaire.

Enfin, la Société de Biologie, à laquelle il s'est consacré avec passion lors de sa retraite, doit aussi beaucoup à Jacques Polonovski. Fondée au XIX ${ }^{\mathrm{e}}$ siècle par Charles Robin et Claude Bernard, à une époque où les chercheurs en biologie formaient un cénacle restreint, capables de se réunir une fois par semaine et de discuter leurs résultats courants, la Société de Biologie n'avait plus au XX $\mathrm{XX}^{\mathrm{e}}$ siècle un format adapté à l'essor formidable de la recherche en biologie et était tombée en déshérence, malgré l'habitude des «patrons » d'y déléguer leurs jeunes élèves y faire leurs premières communications, ce qui fut effectivement notre cas et notre premier souvenir de réunion scientifique.

Jacques Polonovski eut l'idée et l'énergie de transformer la Société en un observatoire de la recherche biologique en France, en créant des séances thématiques mensuelles, animées par les meilleurs spécialistes. Il fut le dernier président (1991-1992) sous le long règne (1953-1992) du secrétaire général Jean ROCHE, avant de lui succéder à cette fonction (1992-1998). Nous eûmes l'un et l'autre l'honneur et le plaisir de l'y seconder et de le remplacer à ces postes. Cette collaboration nous a permis de constater son caractère égal mais obstiné malgré la difficulté des tâches, et surtout d'apprécier ses qualités humaines. Homme discret et modeste, presque timide, catholique serein et engagé, Jacques Polonovski savait attirer l'attachement et l'affection de ses collaborateurs comme de ses collègues.

La Société de Biologie, consciente de sa dette envers cette grande figure du monde universitaire, s'associe à la peine de son épouse, de sa famille et de tous ceux qui ont eu le privilège d'approcher cet homme exquis et chaleureux. 